\section{Admission Rates and Lithium}

SIR: The argument seems to be about the 'efficacy' and perhaps the 'efficiency' of this drug (Journal, February 1987, 150, 264-265). We would like to add further data to this discussion.

Recently, we examined infradian rhythms of mood and allied factors in out-patients with major affective disorders and matched healthy controls (Eastwood et al, 1985). Each person was interviewed with the Schedule for Affective Disorders and Schizophrenia, and the index group had to meet the Research Diagnostic Criteria. A sub-group from this study consisted of 11 patients who had been on lithium for an average of seven years, and these were contrasted with 22 healthy controls. These patients were treated independently by other psychiatrists and took only lithium during the study period. Patients, all of whom had bipolar affective disorders, and controls completed visual analogue scales daily (Eastwood $e t$ al, 1984) for 14 months. The mood ratings for index and control groups were compared using the means, standard deviations, and delta squares. The variability of mood, using chi-square analysis, was significantly skewed towards zero in the lithium group, compared with controls, for the standard deviations and delta squares.

So cases of bipolar affective disorder had less mood variability over 14 months on lithium than normal controls. The Folstein et al (1982) short-term finding in this regard (one month) is more than confirmed. Our study speaks to efficacy, in some cases, but says nothing about efficiency. Elsewhere we have shown that prescribing is not completely efficient, since in Toronto approximately two-thirds of patients with bipolar affective disorders receive lithium and two-thirds of those on lithium have bipolar affective disorders (Eastwood et al, 1981).

The very effectiveness of lithium could create problems by making patients overly controlled, without spontaneity and creativity (Jamison et al, 1979), and cause them to stop taking it. This would fit with Kendell's idea of withdrawal causing relapse and ever more mania. How ironic if patients' noncompliance in this disruptive illness was a function of effectiveness of treatment.

M. R. EASTWOOD

Clarke Institute of Psychiatry

250 College Street

Toronto

Ontario MST IR8

\section{References}

EAstwood, M. R., Stiasny, S. \& Tice, S. (1981) Estimates of the prevalence of bipolar affoctive disorder: methods based on treatment with lithium. Acta Psychiatrica Scandinavica, 63, 83-90.

EAstwood, M. R., Whitton, J. L. \& Kramer, P. M. (1984) A brief instrument for longitudinal monitoring of mood states. Psychiatry Research, 35, 773-783.
Eastwood, M. R., Whitton, J. L., Kramer, P. M. \& Peter, A. M. (1985) Infradian thythms: a comparison of affective disorders and normal persons. Archives of General Psychiatry. 42, 295-299.

Folstein, M. F., DePaulo, J. R. \& TRIPP, K. (1982) Unusual mood stability in patients taking lithium. British Journal of Psychiatry. 140, 188-190.

Jamison, K. R., Gerner, R. H. \& Goodwin, F. K. (1979) Patient and physician attitudes toward lithium. Archives of General Psychiatry, 36, 866-869.

\section{Psychiatric Morbidity and the Mentally Handicapped}

SIR: Day's (1985) reported morbidity of $30 \%$ and $20 \%$ for psychiatric disorder in mentally handicapped patients of a mental handicap hospital and a day hospital respectively, may be equally valid with respect to mentally handicapped out-patients.

In a random analysis of one year's attendance of mentally handicapped patients at a small community-based mental handicap out-patient clinic (January-December, 1982) I found that 19\% of the 115 out-patients seen were suffering from a significant mental illness: depressive illness 10 (i.e. manic depressive 2 , agitated depression 6 , depression with epilepsy 1, depression after brain tumour 1); schizophrenia 9 (i.e. paranoid 6, hebephrenic 2, simple 1); and severe personality disorders 3 . This excludes behaviour disorders which were present in $36 \%$. Diagnoses were made on the basis of a comprehensive history (from patients' relatives, social workers and community nurses), appropriate clinical criteria and, in doubtful cases, by admission for assessment, observation, and follow-up.

Sturmey's claim (Journal, February 1987, 150, 270) that the behaviour problems due to painful physical conditions (abscesses, nasal infection, etc.), learned behaviour, and anti-social behaviour (wandering, stealing, etc.) may inflate the serious psychiatric morbidity in the mentally handicapped is not borne out by the many well-conducted studies in this field. In fact, most of these studies include major psychiatric illnesses such as schizophrenia, affective disorder, and personality disorder, and exclude the behaviour problems which he mentions.

The diagnosis of major psychiatric illness in mentally handicapped patients has always been difficult, as the signs and symptoms of the illness may be masked by the behavioural expression, as illustrated below.

Case report: A young severely mentally handicapped patient attending a special needs unit expressed his mood changes in a regular pattern of periods of overactivity, agitation, aggression, sleeplessness, and exhaustion, which resulted in loss of weight, chest infections, etc. (hypomanic phase), alternating, after a brief period of stability on medication, with phases of withdrawal, irritability, loss of his usual sociability, and tearfulness (depressive phase). This 
cycle lasted between 9 and 15 months. His response to lithium therapy was remarkable, and showed in his physical and emotional well-being, as well as in improvement in his learning, social and personal skills.

I hope that Sturmey is not attempting to minimise the importance of the recognition of treatable major mental illness in mentally handicapped patients, many of whom have suffered greatly in the past from the lack of appropriate treatment (mainly psychotropic medication) simply because their mental disorders were unrecognised.

Llwyneryr Hospital

T. HARI SINGH Morriston

Swansea

\section{Reference}

DAY, K. (1985) Psychiatric disorder in the middle-aged and elderly mentally handicapped. British Journal of Psychiatry, 147, $660-667$

Is the Positive-Negative Distinction in Schizophrenia Valid?

SIR: The longitudinal study by Johnstone et al (Journal, January 1987, 150, 60-64) raises questions on the validity of the concept of positive and negative symptoms in schizophrenia. Their original proposition that this dichotomy represents different pathogenic mechanisms had been predicated largely on the assumption that positive symptoms are treatment responsive, and hence reversible, whereas negative symptoms are not. The current findings indicate, however, that positive symptoms are not uniformly treatment responsive and that negative symptoms are not universally immutable.

Our longitudinal studies of schizophrenia also challenge the validity of the positive-negative model as originally proposed, and suggest that other dimensions, notably chronicity of illness and diagnostic sub-type, may interact with this construct. In a two year follow-up of young patients with acute schizophrenia (Lindenmayer et al, 1986), both positive and negative syndromes proved longitudinally unstable and showed changing patterns of external correlates over time. Contrary to assumptions from studies in the chronic phase, it was the base-line negative rather than positive presentation which anticipated a favourable outcome. A cross-sectional survey of 134 patients with schizophrenia classified as acute (up to 2 years history of illness), chronic (3-10 years), and long-term chronic (over 10 years) similarly suggested that an early negative syndrome carries a better prognosis. In patients with acute schizophrenia such a profile was associated with depressive and atypical catatonic features as well as absence of psychosis in first-degree relatives (Kay et al, 1986). Thus, the positive-negative dichotomy may have a quite different clinical significance in the acute phase of psychosis to that in the chronic phase.

Recent pharmacological analysis has suggested, moreover, that the positive-negative classification delineates homogeneous psychobiological sub-types in schizophrenia only if considered along with diagnostic sub-type (Singh et al, 1987). The addition of anticholinergic drugs to neuroleptic treatment in a mixed population of patients with acute, subacute and chronic schizophrenia produced a significant worsening of positive symptoms overall, but this occurred mainly among patients with catatonic symptoms. In paranoid patients, to the contrary, it was the negative symptoms that worsened significantly, whereas in hebephrenic patients neither syndrome was significantly affected. Cumulatively, our results suggested that the natures of positive and negative syndromes vary according to chronicity and subtype of schizophrenia, and perhaps only in the chronic patients and the non-paranoid forms of illness is the present concept of the positive-negative dichotomy applicable.

The data from Johnstone's group underscore qualifications on the generality of the construct. They observed that delusions - a cardinal feature of paranoid schizophrenia-were significantly more prevalent in neuroleptic-treated than neurolepticfree patients with schizophrenia, as were also symptoms of anxiety and depression. The latter finding may be understood within the context of a postneuroleptic emergence of dysphoria and elevation of tonic autonomic arousal, which characterises only non-paranoid patients and portends poor therapeutic response (Singh \& Kay, 1979). Furthermore, our work has suggested that non-paranoid neurolepticresistant patients with schizophrenia may be distinguished from others by clinical worsening under blind wheat gluten challenge (Singh, 1978; Singh \& Kay, 1984). Research on the psychobiology and course of schizophrenia, therefore, casts doubt on the sufficiency of the positive-negative model. Study of its interaction with diagnostic sub-type and phase of illness, on the other hand, may offer new insights not afforded by examining any of these dimensions separately.

\section{Schizophrenic Research Program}

Man Mohan Singh

901 Southwind Road

Springfield, Illinois 62703, USA

Bronx Psychiatric Center

Stanley R. KaY

1500 Waters Place

Bronx, New York 10461, USA 